\title{
Correction to: Efficacy and safety of PEGylated exenatide injection (PB-119) in treatment-naive type 2 diabetes mellitus patients: a Phase II randomised, double-blind, parallel, placebo-controlled study
}

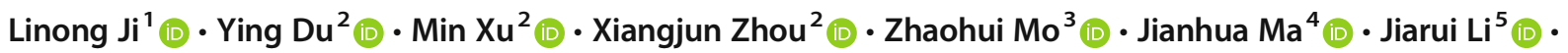

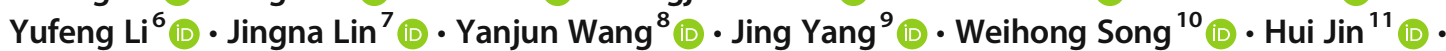 \\ Shuguang Pang ${ }^{12}$ D $\cdot$ Hui Liu ${ }^{13}$ (D) Ping Li ${ }^{14}$ (D) Jie Liu ${ }^{15}$ (D) Minxiu Yao ${ }^{16}$ (D) Wenhui Li ${ }^{17}$ (D) Xiaohong Jiang ${ }^{18}$ (D) \\ Feixia Shen ${ }^{19}$ (D) Houfa Geng ${ }^{20}$ (D) $\cdot$ Haifeng Zhou ${ }^{21}$ (D) $\cdot$ Jianmin Ran $^{22}$ (D) Minxiang Lei ${ }^{23}$ (D) $\cdot$ Yinghong Du $^{24}$ (D) $\cdot$ \\ Shandong $\mathrm{Ye}^{25}$ (D) - Qingbo Guan ${ }^{26}$ (D) - Wenshan $\mathrm{Lv}^{27}$ (D) - Huiwen $\operatorname{Tan}^{28}$ (D) Tao Chen $^{28}$ (D) Jinkui Yang ${ }^{29}$ (D) \\ Guijun Qin $^{30}$ (D) Shiyun $\mathrm{Li}^{31}$ (D) Lei Chen ${ }^{32}$ (D)
}

(C) The Author(s) 2021

\section{Correction to: Diabetologia} https://doi.org/10.1007/s00125-021-05392-9

The following sentence in the Discussion was inappropriately changed to a definite statement during editing: 'Considering the mechanism of action of PB-119, which is similar to exenatide QW, PB-119 also showed superior efficacy compared with available OADs.'

The journal would like to revert back to the authors' original phrasing as follows:

'Considering the mechanism of action of PB-119, which is similar to exenatide QW, PB-119 could also reveal superior efficacy in comparison with available OADs.'
The original article has been corrected.

Open Access This article is licensed under a Creative Commons Attribution 4.0 International License, which permits use, sharing, adaptation, distribution and reproduction in any medium or format, as long as you give appropriate credit to the original author(s) and the source, provide a link to the Creative Commons licence, and indicate if changes were made. The images or other third party material in this article are included in the article's Creative Commons licence, unless indicated otherwise in a credit line to the material. If material is not included in the article's Creative Commons licence and your intended use is not permitted by statutory regulation or exceeds the permitted use, you will need to obtain permission directly from the copyright holder. To view a copy of this licence, visit http://creativecommons.org/licenses/by/4.0/.

Publisher's note Springer Nature remains neutral with regard to jurisdictional claims in published maps and institutional affiliations.

The online version of the original article can be found at https://doi.org/ $10.1007 / \mathrm{s} 00125-021-05392-9$

Linong Ji

jiln@bjmu.edu.cn

Extended author information available on the last page of the article 


\section{Affiliations}

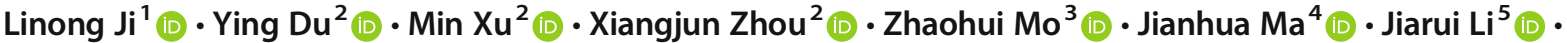

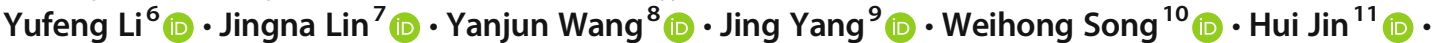 Shuguang Pang ${ }^{12}$ (D) Hui Liu ${ }^{13}$ (D) $\cdot$ Ping $\mathrm{Li}^{14}$ (D) $\cdot \mathrm{Jie} \mathrm{Liu}^{15}$ (D) Minxiu Yao ${ }^{16}$ (D) $\cdot$ Wenhui Li ${ }^{17}$ (D) Xiaohong Jiang $^{18}$ (D)

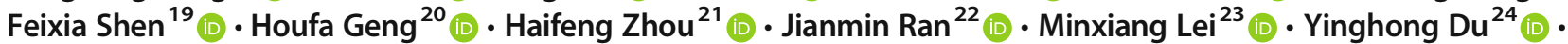 Shandong $\mathrm{Ye}^{25}$ (D) $\cdot$ Qingbo Guan ${ }^{26}$ (D) $\cdot$ Wenshan $\mathrm{Lv}^{27}$ (D) $\cdot$ Huiwen $\operatorname{Tan}^{28}$ (I) $\cdot$ Tao Chen $^{28}$ (D) - Jinkui Yang ${ }^{29}$ (D) Guijun Qin ${ }^{30}$ (D) Shiyun $\mathrm{Li}^{31}$ (D) $\cdot$ Lei Chen ${ }^{32}$ (I)}

1 Department of Endocrinology, Peking University People's Hospital, Beijing, China

2 PegBio Co., Ltd, Suzhou, China

3 Department of Endocrinology, The Third Xiangya Hospital of Central South University, Changsha, China

4 Department of Endocrinology, Nanjing First Hospital, Nanjing, China

5 The Third Endocrinology Department, Cangzhou Central Hospital, Cangzhou, China

6 Department of Endocrinology, Beijing Pinggu Hospital, Beijing, China

7 Department of Endocrinology, Tianjin People's Hospital, Tianjin, China

8 Department of Endocrinology, The Second Hospital of Jilin University, Changchun, China

9 Department of Endocrinology, First Hospital of Shanxi Medical University, Taiyuan, China

10 Department of Endocrinology and Diabetes, Chenzhou No 1 People's Hospital, Chenzhou, China

11 Department of Endocrinology, Zhongda Hospital Southeast University, Nanjing, China

12 Department of Endocrinology, Jinan Central Hospital, Jinan, China

13 Department of Endocrinology, Luoyang Central Hospital, Luoyang, China

14 Department of Endocrinology, Yuncheng Central Hospital, Yuncheng, China

15 Department of Endocrinology, The First Affiliated Hospital of Henan University of Science and Technology, Henan, China

16 Department of Endocrinology, Qingdao Central Hospital, Qingdao, China

17 Department of Endocrinology, Beijing Union Medical College Hospital, Beijing, China
18 Department of Endocrinology, The First People's Hospital of Changzhou, Changzhou, China

19 Department of Endocrinology, The First Affiliated Hospital of Wenzhou Medical University, Wenzhou, China

20 Department of Endocrinology, Xuzhou Central Hospital, Xuzhou, China

21 Department of Endocrinology, The First People's Hospital, Changde, China

22 Department of Endocrinology, Guangzhou Red Cross Hospital, Guangzhou, China

23 Department of Endocrinology, Xiangya Hospital Central South University, Changsha, China

24 Department of Endocrinology, Guangzhou Panyu Central Hospital, Guangzhou, China

25 Department of Endocrinology, Anhui Provincial Hospital, Hefei, China

26 Department of Endocrinology, Shandong Provincial Hospital, Jinan, China

27 Department of Endocrinology, The Affiliated Hospital of Qingdao University, Qingdao, China

28 Department of Endocrinology, West China Hospital Sichuan University, Sichuan, China

29 Department of Endocrinology, Beijing Tongren Hospital, CMU, Beijing, China

30 Department of Endocrinology, The First Affiliated Hospital of Zhengzhou University, Henan, China

31 Department of Endocrinology, Affiliated Hospital \& Clinical Medical College of Chengdu University, Chengdu, China

32 Department of Endocrinology, Suzhou Municipal Hospital, Suzhou, China 